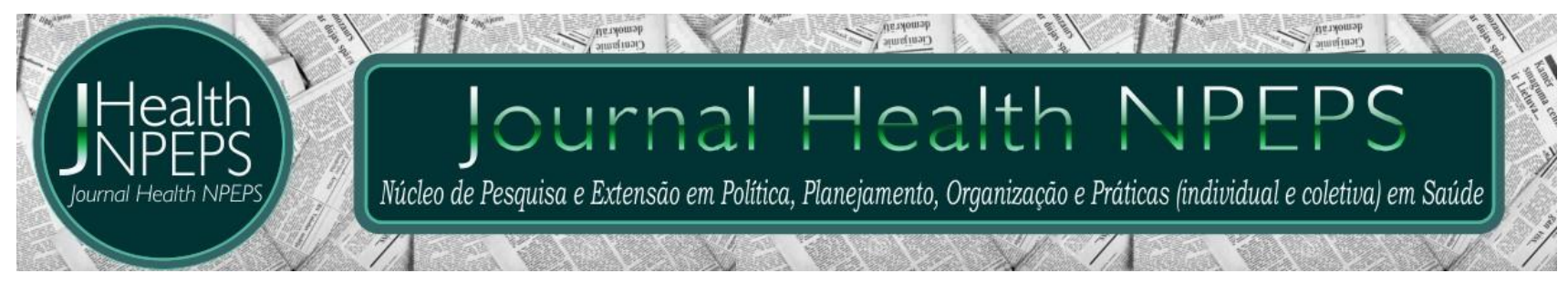

http://dx.doi.org/10.30681/252610104585

ARTIGO ORIGINAL

\title{
Comunicação do diagnóstico de câncer colorretal à pessoa e família e/ou cuidador
}

\author{
Communication of colorretal cancer diagnosis to the person and family and/or \\ caregiver
}
Comunicación del diagnóstico de cáncer colorretal a la persona y la familia y/o cuidador

\begin{abstract}
Michele Cristiene Nachtigall Barboza1 ${ }^{1}$, Rosani Manfrin Muniz ${ }^{2}$, Raquel Cagliari ${ }^{3}$, Juliana Amaral Rockembach ${ }^{4}$, Sandy Alves Vasconcellos ${ }^{5}$, Letícia Valente Dias ${ }^{6}$
\end{abstract}

\section{RESUMO}

Objetivo: conhecer o impacto da comunicação do diagnóstico de câncer colorretal à pessoa e sua família e/ou cuidador. Método: pesquisa qualitativa, realizada por meio de entrevistas, junto a 11 pessoas com câncer colorretal e 12 familiares, de maio a julho de 2017, em um município do Rio Grande do Sul, Brasil. Os dados foram organizados e codificados no software Webqda e submetidos à análise temática. Resultados: emergiram três categorias, problemas na habilidade de comunicar más notícias; a terminologia técnica na comunicação do diagnóstico de câncer colorretal; conflitos na comunicação do diagnóstico de câncer colorretal às pessoas idosas. Considerações finais: destaca-se a necessidade de qualificação profissional em relação à linguagem utilizada e formas de expressão, na perspectiva de estabelecê-la de modo mais empático, e consequentemente promover maior segurança e confiança nas relações entre pessoas doentes, família e profissionais de saúde.

Descritores: Neoplasias Colorretais; Comunicação; Família.

\section{ABSTRACT}

\footnotetext{
${ }^{1}$ Enfermeira. Doutora em Ciências. Universidade Federal de Pelotas. Pelotas, Rio Grande do Sul, Brasil. E-mail: michelenachtigall@yahoo.com.br ORCID ID: http://orcid.org/0000-0003-1489-5813 Autor para correspondência Endereço: Barão de Santa Tecla 788, apto 301. Pelotas, Rio Grande do Sul, Brasil. CEP: 96010-140.

${ }^{2}$ Enfermeira. Doutora em Enfermagem. Universidade Federal de Pelotas. Pelotas, Rio Grande do Sul, Brasil. E-mail: romaniz@terra.com.br ORCID ID: http://orcid.org/0000-0002-5642-7842

${ }^{3}$ Enfermeira. Universidade Federal de Pelotas. Pelotas, Rio Grande do Sul, Brasil. E-mail: cagliariraquel01@gmail.com ORCID ID: http://orcid.org/0000-0002-5309-961X

${ }^{4}$ Enfermeira. Mestre em Ciências. Universidade Federal de Pelotas. Pelotas, Rio Grande do Sul, Brasil. E-mail: ju.rockembach@hotmail.com ORCID ID: http://orcid.org/0000-0002-0693-8775

${ }^{5}$ Enfermeira. Especialista em Oncologia. Universidade Federal de Pelotas. Pelotas, Rio Grande do Sul, Brasil. E-mail: sandyalvesvasconcellos@hotmail.com ORCID ID: https://orcid.org/0000-0002-0497-078X

${ }^{6}$ Enfermeira. Mestre em Ciências. Universidade Federal de Pelotas. Pelotas, Rio Grande do Sul, Brasil. E-mail: leticia diazz@hotmail.com ORCID ID: http://orcid.org/0000-0002-5812-4079
} 
Objective: to know the impact of communicating the diagnosis of colorectal cancer to the person and their family and/ or caregiver. Method: qualitative research, conducted through interviews, with 11 people with colorectal cancer and 12 family members, from May to July 2017, in a municipality in Rio Grande do Sul, Brazil. The data were organized and coded in the Webqda software and submitted to thematic analysis. Results: three categories emerged, problems with the ability to communicate bad news; technical terminology in communicating the diagnosis of colorectal cancer; conflicts in communicating colorectal cancer diagnosis to the elderly. Final considerations: the need for professional qualification in relation to the language used and forms of expression, in the perspective of establishing it in a more empathic way, and consequently promoting greater security and trust in the relationships between sick people, family and health professionals is highlighted.

Descriptors: Colorectal Neoplasms; Communication; Family.

\section{RESUMEN}

Objetivo: conocer el impacto de comunicar el diagnóstico de cáncer colorrectal a la persona y su familia y/o cuidador. Método: investigación cualitativa, realizada a través de entrevistas, con 11 personas con cáncer colorrectal y 12 familiares, de mayo a julio de 2017, en un municipio de Rio Grande do Sul, Brasil. Los datos se organizaron y codificaron en el software Webqda y se sometieron a análisis temático. Resultados: surgieron tres categorías, problemas con la capacidad de comunicar malas notícias; terminología técnica para comunicar el diagnóstico de cáncer colorrectal; conflictos en la comunicación del diagnóstico de cáncer colorrectal a los ancianos. Consideraciones finales: se destaca la necesidad de cualificación profesional en relación con el lenguaje utilizado y las formas de expresión, en la perspectiva de establecerlo de una manera más empática, y en consecuencia promover una mayor seguridad y confianza en las relaciones entre los enfermos, la familia y los profesionales de la salud.

Descriptores: Neoplasias Colorrectales; Comunicación; Familia.

\section{INTRODUÇÃO}

O câncer é considerado um problema de saúde pública de âmbito mundial. Para 2025 é esperado o surgimento de mais de 20 milhões de novos casos. Destes, o câncer colorretal (CCR) se destaca como a terceira neoplasia mais incidente ${ }^{1}$. No Brasil, as estimativas dessa neoplasia apontam para a ocorrência de mais de 40 mil novos casos no ano de 2020. Em 2018 esteve entre as três primeiras causas de morte por câncer, resultando na mortalidade de mais de 19 mil pessoas ${ }^{2}$.

O diagnóstico de uma doença grave, com alta morbidade e mortalidade, como o CCR, acarreta sofrimento físico e emocional à pessoa doente e seus familiares e/ou cuidadores. Esse processo é vivenciado como um momento de angústia, rodeado por estigmas sociais que estão associados a um tratamento mutilador, doloroso e letal ${ }^{3}$.

Nessa perspectiva, comunicar más notícias, como o diagnóstico de Journal Health NPEPS. 2020 jul-dez; 5(2):226-239. 
câncer, torna-se um evento delicado, e por isso, a forma como é passada a informação é fundamental no intuito de minimizar o sofrimento atribuído a essa malignidade. Neste intuito, a qualidade da comunicação por parte do profissional deve estar embasada em um atendimento de qualidade, conferindo apoio emocional, orientações, auxílio e confiança para lidar com as adversidades impostas pelo adoecimento ${ }^{4}$.

Desse modo, a realização de uma capacitação para desenvolver habilidade de comunicação a profissionais de saúde, que trabalham no tratamento do câncer, podem resultar em melhorias na interlocução, a fim de promover uma relação mais empática, oferecendo apoio frente às emoções dos doentes ${ }^{4}$.

Assim, profissionais podem proporcionar o cuidado de qualidade à pessoa doente e seus familiares, ao transmitir más notícias, pois quando ocorre de maneira eficaz, atua como uma ferramenta que opera na resolução de anseios e preserva a autonomia de todos envolvidos. Para tanto, é necessário empatia por parte do profissional, de modo que a comunicação ocorra de forma esclarecedora e que atenda as expectativas das pessoas sob seus cuidados ${ }^{4}$.

Nesse sentido, o presente estudo busca responder a seguinte questão de pesquisa: qual 0 impacto da comunicação do diagnóstico à pessoa com CCR e seu familiar e/ou cuidador? Com isso, a pesquisa tem por objetivo conhecer o impacto da comunicação do diagnóstico de CCR à pessoa e sua família e/ou cuidador.

\section{MÉTODO}

Trata-se de estudo descritivo, de abordagem qualitativa, 0 qual sua elaboração atendeu ao checklist da Consolidated criteria for reporting qualitative research (COREQ) ${ }^{5}$. Este estudo foi desenvolvido em dois cenários, um deles foi o Programa de Assistência do Estomizado e Incontinente (PAEI), serviço da secretaria municipal de saúde de um município do Rio Grande do Sul, a fim de conhecer e selecionar os possíveis participantes do estudo. E o outro no domicílio das pessoas com câncer e seus familiares e/ou cuidadores, na perspectiva de estabelecer um vínculo mediante o conforto que o domicílio proporciona.

No PAEI foi possível selecionar alguns dos participantes, uma vez que os mesmos iam até o serviço para consultas ou busca de materiais (bolsas de estomias e adjuvantes) e ali eram 
convidados. Contudo, alguns convites foram realizados via telefone, com três recusas, justificadas pelo avanço da doença, pela falta de tempo, ou simplesmente por não querer falar sobre o assunto, porém seis participantes aceitaram participar de imediato. Os contatos telefônicos foram obtidos mediante os prontuários dos pacientes do serviço.

Participaram do estudo as pessoas que estavam em consonância com os seguintes critérios de inclusão: diagnóstico de CCR e assistido pelo PAEI há pelo menos 12 meses (diagnóstico a partir de maio de 2016); familiar sugerido pela pessoa que teve CCR assistida pelo PAEI; ser indicado pela enfermeira do PAEI; ter ciência da doença e condição de saúde; ser maior de 18 anos; ter capacidade de ouvir e falar sem dificuldade. Como critérios de exclusão: não foi elegível o participante que apresentasse limitação comunicativa, cognitiva ou que não tivesse clareza de seu diagnóstico. Optou-se por selecionar pessoas com até 12 meses de diagnóstico, a fim de garantir lembranças recentes desse período de vida, durante o período de coleta, bem como a indicação da enfermeira do programa, pelo conhecimento prévio da história clinica dos pacientes e assim garantiria a agilidade no processo de coleta. Utilizou-se o critério de saturação teórica, o qual ocorre à suspensão de inclusão de novos participantes devido à repetição das informações ou poucas informações relevantes ${ }^{6}$.

A coleta dos dados foi realizada entre os meses de maio a julho de 2017. A técnica para a coleta dos dados foi de entrevista em profundidade. As questões que nortearam as entrevistas foram: "fale-me sobre a doença desde o início e o que fez para cuidar da saúde antes e após ter a doença”, "fale-me sobre sua vida com a colostomia", "Como foi sua participação no cuidado à saúde, enquanto familiar e/ou cuidador?". Estas questões foram utilizadas para dar início à entrevista e conforme necessidade, outras foram realizadas, a fim de abarcar a totalidade do fenômeno. Todos os participantes foram entrevistados por no mínimo duas vezes para garantir o aprofundamento das informações fornecidas e obter as correções das transcrições dos dados coletados. Dois participantes necessitaram de uma terceira visita, uma vez que a familiar/cuidador não estava disponível naquele momento para ser entrevistada.

Todas as informações foram 
gravadas em mídia digital e transcritas na íntegra. O tempo de gravação foi de $2 \mathrm{~h}$ e $22 \mathrm{~m}$ a mais longa e de 40 minutos a mais curta. Entretanto, o tempo médio de cada encontro foi de quatro horas, pois havia uma conversa informal pré e pós-gravação da entrevista. Após a transcrição, os dados foram organizados e codificados no software de acesso online, chamado webQDA. Este software possibilita ao investigador editar, visualizar, interligar e organizar documentos, bem como criar categorias, codificar, controlar, filtrar, efetuar pesquisas e questionar os dados, na perspectiva de responder às suas questões de investigação e objetivo da pesquisa ${ }^{7}$.

Os dados foram analisados de acordo com a análise temática ${ }^{8}$ que compreende seis etapas: A primeira etapa foi à familiarização com os dados coletados, sendo necessário mergulhar nos dados para conhecer o conteúdo em profundidade, ou seja, as entrevistas, que foram transcritas na íntegra. $\mathrm{Na}$ segunda fase ocorreu a geração de códigos iniciais, gerando uma lista inicial de ideias interessantes, sendo eles agrupados conforme relevância e inseridos no software webQDA, iniciando os códigos árvore e após criando subcódigos. A terceira fase consistiu na procura por temas, sendo identificados por um conjunto de dados agrupados em códigos potenciais. $\mathrm{Na}$ quarta fase se realizou a revisão dos temas, a fim de confirmar realmente sua importância e relevância. $\mathrm{Na}$ quinta fase se estabeleceu a definição e nomeação dos temas. E finalmente a sexta e última fase se produziu o relatório, elaborado a partir do conjunto dos temas, envolvendo a análise final dos dados selecionados.

A análise dos dados possibilitou emergir três categorias: "Problemas na habilidade de comunicar más notícias"; “A terminologia técnica na comunicação do diagnóstico de CCR"; "Conflitos na comunicação do diagnóstico de CCR a pessoas idosas".

Os participantes com CCR e familiares foram identificados mediante nomes fictícios escolhidos pelos mesmos, acrescido da idade. Em relação ao familiar, este foi especificado na sua posição/papel (esposo/a, pai ou mãe, entre outros). Antes de iniciar a pesquisa foi realizado contato inicial e convite aos participantes do estudo. Foi apresentada a pesquisa, seus objetivos, possíveis riscos e benefícios, bem como dados contidos no Termo de Consentimento Livre e Esclarecido (TCLE). Todos os participantes foram 
convidados para participarem da apresentação dos dados na banca de defesa do estudo, a qual dois participantes se fizeram presentes.

Foram respeitados os preceitos éticos definidos pela resolução $\mathrm{n}^{\circ}$. 466/12 do Conselho Nacional de Saúde do Ministério da Saúde, que regulamenta pesquisas envolvendo seres humanos. A pesquisa teve aprovação em um Comitê de Ética e Pesquisa, com CAAE n. 67857417.0.0000.5337 e parecer $n$. 2.063.328.

\section{RESULTADOS}

Participaram do estudo 23 pessoas, sendo que 11 eram pessoas que tiveram CCR e 12 eram familiares das mesmas. Entre os participantes com CCR prevaleceu o sexo masculino $(n=8)$ na faixa etária dos 31 a 82 anos de idade. A maioria dos homens com CCR indicou como familiar participante do estudo a esposa, enquanto as mulheres com CCR indicaram a filha. Já entre os participantes familiares, prevaleceu 0 sexo feminino $(n=11)$, com idades entre 21 e 77 anos.

Quanto ao sítio anatômico acometido pelo CCR, houve neoplasia de reto $(n=4)$, neoplasia de sigmoide $(n=3)$, com neoplasia maligna de retossigmóide $(\mathrm{n}=2)$ a neoplasia de cólon descendente $(n=1)$ e obstrução colônica tumoral $(n=1)$. Somente um dos participantes possuía estomia no íleo, os demais no cólon. A modalidade terapêutica utilizada para tratamento em todos os participantes foi à cirurgia, sendo que alguns também receberam a quimioterapia como tratamento coadjuvante $(n=7)$. Durante o período de coleta três participantes ainda aguardavam pelo serviço para iniciar o tratamento antineoplásico, seja a radioterapia ou a quimioterapia.

\section{Problemas na habilidade de comunicar} más notícias

O processo de comunicação do diagnóstico de câncer pode ser considerado traumático ou assustador a depender da forma como as palavras são explicitadas ou a maneira como as condiçõos de adoecimento são expostas pelos profissionais de saúde. Desse modo, as falas abaixo apresentam problemas relacionados à capacidade técnica dos profissionais de saúde na comunicação do diagnóstico e possibilidades terapêuticas.

Ele (médico) me deu a notícia na lata, ele simplesmente abriu o exame, abriu o exame e disse seu Mário o Sr. tá com câncer e é 
maligno. E eu digo ah é? [...] Aí ele me explicou direitinho. $O$ senhor tem dois caminhos aqui ó, ou o senhor faz a cirurgia e liquida com isso aí, e se não fizer o senhor vai sofrer. (Mário, 82 anos)

Ele (médico) disse, nas condições que ela tá não sei se sai da mesa, e se saí não sei se dá seis meses, falou assim na minha cara. A gente é obrigado a operar ela, mas nas condições que ela tá, não sei se sai da mesa e se sair não te dou 6 meses. (Cláudio, esposo da Enilda, 56 anos)

Eu levei o pai pra fazer um exame e a doutora chegou e disse o teu pai tem câncer e se ele não se cuidar, ele vai morrer. Nessas palavras, bem assim, nessas palavras com toda essa delicadeza. Sabe eu não tenho nada de bom pra te dizer, o que eu tenho pra te dizer que se tu não cuidar amanhã ou depois ele vai morrer, bem assim ela disse pra mim. (Gertrudes, filha de João, 25 anos)

Nas narrativas dos participantes percebe que na comunicação do diagnóstico do CCR os profissionais utilizam palavras como "não tem condições de sair da mesa” ou "ele vai morrer". Desse modo, o processo de comunicação do diagnóstico com os familiares pode ser considerado traumático ou assustador, devido à forma em que as palavras foram explicitadas ou a maneira que expuseram a condição de adoecimento.

Associar ao sofrimento quando se recebe o diagnóstico de CCR pode não ser a melhor estratégia de comunicar a situação de adoecimento, especialmente em um momento que as pessoas se encontram fragilizadas emocionalmente. Tal atitude evidencia problemas na habilidade em comunicar más notícias feitas pelos profissionais de saúde.

\section{A terminologia técnica na comunicação do diagnóstico de câncer colorretal}

A utilização de linguagem técnica ou científica foi identificada pelos pacientes e seus familiares e/ou cuidadores como um fator prejudicial à comunicação com os profissionais de saúde. Em um dos fragmentos abaixo o termo utilizado para descrever a condição de saúde de uma das participantes fez com que a mesma fosse submetida a procedimentos invasivos sem a total compreensão de sua doença.

Quando eu fiz a cirurgia eles tiraram tudo pra fora pra fazer a limpeza [...]. Depois que limparam tudo que descobriram que tava com a neoplasia. [...] mas fui saber do câncer lá no paliteiro (ambulatório) com a oncologista, porque lá no hospital falaram para eles (familiares) em seguida que me operaram, que eu tinha neoplasia de reto e fígado, não foi câncer que eles falaram. (Enilda, 56 anos)

E pólipo, esse aí que é o danado, foi desse aí que surgiu o meu tumor. [...] Eu custei a entender o que era isso, quando o doutor falou na consulta. Aí pedi para a esposa do meu enteado pesquisar na internet e me explicar. Ele começa pequeno, de grau pequeno depois vai até 5 né, o meu estava no 2 né, e o meu era (pausa) como é que se chama (pausa) aquele que avança, invasor, porque tem uns que são mais 
calminhos né, o meu era invasor quer dizer, que ele ia crescer ligeiro. (Mário, 82 anos)

Foi horrível, foi pra mim, quando eu soube que ele tava assim, foi uma baque muito grande né. [...] Ela (médica) falou primeiro que era por causa de pólipos, mas nós nem sabíamos o que era isso. Mas foi quando falou a palavra câncer aí que foi uma batida muito grande. (Clara, esposa de Gabriel, 35 anos)

A utilização de termos incompreensíveis a leigos mediante uma comunicação com linguagem técnica ou científica interfere na compreensão sobre a condição de saúde de alguns pacientes e seus familiares e/ou cuidadores. Desse modo, é necessário que a comunicação seja efetiva, clara e empática para que as pessoas se sintam acolhidas pelos profissionais, em especial por estarem vulneráveis e emocionalmente desorganizadas diante de uma doença grave, como o câncer.

Entende-se que estabelecer uma comunicação afetiva com as pessoas em ambiente hospitalar é uma atividade complicada para os profissionais de saúde, principalmente diante de cirurgia em condições complexas e com a possibilidade eminente de morte. Entretanto, compreender com clareza o diagnóstico da doença dias após 0 procedimento cirúrgico, na consulta com o oncologista, pode ser considerado uma falha importante na comunicação entre Journal Health NPEPS. 2020 jul-dez; 5(2):226-239. pacientes e profissionais de saúde.

Conflitos na comunicação do
diagnóstico de câncer colorretal a
pessoas idosas

Um aspecto relevante encontrado neste estudo são as particularidades do processo de comunicação entre pessoa doente, familiares e profissionais de saúde quando há o envolvimento de pessoas idosas. As barreiras de comunicação nesses casos poderão estar relacionadas a dificuldades em transmitir informações e orientações, gerando, muitas vezes, mal entendidos. A seguir os participantes apontam a falta de manejo do serviço de saúde ao se deparar com paciente e familiar e/ou cuidador idosos.

\begin{abstract}
Minha filha me disse, amanhã é pro seu Mário ir lá no doutor, mas pediram pra levar um familiar. Eu disse sou eu, sempre vou com ele, eu sou a familiar dele, eu vou, pois sabe que eles ficaram brabos. Ah o médico não gostou, reclamou, reclamou que tinha mandado a secretária ligar pra levar um familiar e foram só dois idosos, velhos. E tinha que ir um jovem com ele, já fiquei braba com eles, velhinho é o pai dele, a mãe dele (risadas). (Gelci, esposa Mário, 77 anos)
\end{abstract}

Ele (médico) reclamou, reclamou mesmo. Não nos respeitou. (Mário, 82 anos)

Ah, eu pensei, eu pensei tanta coisa. Eu já cuidei o pai e a mãe com câncer, então foi horrível e foi terrível, foi um terror. [...] Precisei que me 
ajudassem, não conseguia me acalmar. Eles tiveram que chamar meu filho (Pafúncia, esposa Jeremias, 63 anos).

Receber o diagnóstico de CCR para qualquer pessoa, independentemente da idade ou sexo não é um momento fácil. A própria palavra câncer pode causar um desconforto emocional significativo. Assim, quando o familiar/cuidador é uma pessoa idosa, e ainda possui fragilidades em relação sua saúde, há necessidade de minimizar sofrimentos e não ampliar os conflitos, como observado nas narrativas.

\section{DISCUSSÃO}

Percebe-se que para alguns participantes da pesquisa a comunicação do diagnóstico de CCR nos diferentes serviços de saúde pode ser considerada traumática, em especial por associar o câncer ao sofrimento e pouco tempo de vida ou ainda pela linguagem técnica utilizada.

Muitas vezes, os profissionais que realizam os exames diagnósticos, não são os profissionais responsáveis pelo tratamento e cuidado do paciente, devido a fragmentação dos serviços de saúde. Desse modo, por não terem vínculos estabelecidos como os pacientes os profissionais expressam os Journal Health NPEPS. 2020 jul-dez; 5(2):226-239. diagnósticos de forma mais técnica e sem envolvimento, ou seja, a comunicação acaba sendo objetiva ou direta, o que pode aumentar a ansiedade e medo diante de uma doença estigmatizada9. Entretanto, é importante destacar a necessidade de profissional com habilidades para trabalhar de forma empática com 0 paciente e família e/ou cuidador, na perspectiva de tranquilizar e equalizar essas nuances emocionais diante deste momento complexo.

A comunicação de más notícias necessita ser aplicada mediante capacitação e treinamento entre todos os profissionais envolvidos no contexto do adoecimento, uma vez que a forma utilizada para transmitir este diagnóstico é de extrema importância para promover melhor aceitação e enfrentamento de uma doença grave, como o câncer ${ }^{10}$.

No entendimento de alguns profissionais, a comunicação com os familiares pode ser realizada de maneira direta, uma vez que não são eles que se encontram doentes ${ }^{11}$. Contudo, a família em situação de adoecimento, pode sofrer até mais que a pessoa doente, o que exige mais habilidade do profissional responsável por estabelecer este diagnóstico, destacando que independente de conhecer ou não o 
paciente e família necessita compreender o que estes estão passando e perceber a grandiosidade dos sofrimentos do outro ${ }^{12}$.

Os profissionais ao utilizarem terminologias técnicas necessitam se preocupar em abordar os significados destas terminologias, para assim proporcionar esclarecimento e compreensão do assunto, ou simplesmente não as utilizar, pois nem todos conseguem entendê-las. Dar más notícias as pessoas é uma competência relevante do profissional de saúde, que exige uma abordagem empática e acolhedora, na perspectiva de respeitar o momento vivenciado pelos pacientes, familiares e/ou cuidadores, tornando-se uma ferramenta essencial na assistência de qualidade no contexto de cuidados oncológicos ${ }^{13}$.

A construção de vínculos entre profissionais, pacientes, familiares e/ou cuidadores vai ao encontro de uma comunicação efetiva, o qual estabelece o entendimento e a compreensão das informações, além de tornar os pacientes e familiares mais ativos na trajetória da doença e parceiros na realização de cuidados, bem como contemplar suas dúvidas e as informações consideradas importantes sobre a doença e tratamento realizado ${ }^{14}$.
No entanto, o vínculo não deve ser um fator determinante para a comunicação de más notícias, uma vez que todas as pessoas merecem um atendimento cuidadoso que transmita segurança aos envolvidos.

Considera-se que a fragilidade do familiar e/ou cuidador idoso é justificada pela preocupação do serviço em comunicar uma má notícia, como o diagnóstico do câncer, a outra pessoa igualmente idosa, podendo esta não receber esse pronunciamento facilmente. Todavia, negar a presença de um familiar e/ou cuidador no momento da comunicação, pode acarretar em quebra de confiança, independentemente da idade do familiar e/ou cuidador. A inclusão do acompanhante reflete em uma assistência de saúde mais humanizada. A atenção dada ao familiar e/ou cuidador durante a comunicação de más notícias permite um cuidado centrado no paciente e seu acompanhante, transformando-os em protagonistas do cenário em que estão inseridos ${ }^{15}$.

Comunicar o diagnóstico de câncer exige uma atenção significativa dos profissionais ${ }^{16}$, especialmente quando realizada junto a pessoas idosas, uma vez que muitas podem apresentar capacidade cognitiva prejudicada, e não 
conseguirem alcançar a relação terapêutica necessária nesse processo de comunicação. Nesse contexto, os profissionais de saúde devem estar preparados para estabelecer uma comunicação em saúde efetiva, para um cuidado integralizado, individualizado, com respeito ao contexto e as multidimensões de todos os envolvidos no processo de adoecimento ${ }^{17}$.

Desse modo, considera-se relevante refletir que a comunicação de más notícias no Brasil é feita somente por uma categoria profissional, que pode não estar preparada para estabelecer uma comunicação mais empática e menos indiferente, podendo ser realizada juntamente com uma equipe multiprofissional, o que poderia facilitar a compreensão do processo de adoecimento como um todo. Ademais, ampliar a relação entre outros membros da equipe de saúde seria empoderar e dar autonomia para profissionais com maior sensibilidade e poder de comunicação empática, além de promover uma maior harmonia em equipe $^{18}$.

\section{CONSIDERAÇÕES FINAIS}

Ao buscar conhecer o impacto da comunicação do diagnóstico de câncer à pessoa doente, familiar e/ou cuidador, o presente estudo evidenciou os desafios e as dificuldades atrelados a esse processo. Dentre os principais resultados destacam-se os problemas na habilidade de comunicação de más notícias dos profissionais de saúde, a utilização de terminologia técnica na comunicação do diagnóstico de câncer colorretal e conflitos na comunicação do diagnóstico de câncer colorretal a pessoas idosas.

Assim, a experiência do recebimento do diagnóstico apresentouse de maneira negativa para os participantes, principalmente pela maneira objetiva como a informação foi repassada e as correlações do câncer e ao sofrimento que envolve o diagnóstico desta doença. É notável, portanto, a necessidade de adaptação dos profissionais com relação à linguagem utilizada e formas de expressão, a fim de favorecer a compreensão da situação e estabelecer uma relação de segurança e confiança.

Nesse sentido, a comunicação de más notícias pode ser facilitada com o trabalho de uma equipe multiprofissional capaz de atender a pessoa doente e família e/ou cuidador, nesse momento emocionalmente conturbado. Para tal, habilidades relacionais como a construção de vínculo e a sensibilidade 
podem implicar em maior tranquilidade e redução do sofrimento. Ademais, comunicar más notícias a pessoas idosas exige uma habilidade e cuidado ainda maior da equipe de saúde, a fim de evitar que informações sejam compreendidas de maneira equivocada ou ainda que possam também afetar sua saúde.

Face ao exposto, destaca-se como contribuição deste estudo a necessidade de rever a atenção prestada à pessoa com CCR e família e/ou cuidador. O estudo evidencia o impacto do diagnóstico, e este estende por todo o núcleo familiar, pois os envolvidos não sofrem somente pelo diagnóstico recebido, mas também pela forma como ele é dado e como são acolhidos nos serviços de saúde. Destaca-se ainda que as pessoas ao sofrerem o impacto deste diagnóstico possivelmente ficarão desestabilizadas, desorganizadas e vulneráveis a agravamentos.

Outro aspecto a ser destacado na comunicação de diagnóstico de câncer pode estar associado ao tipo de ensino biomédico e curativista estabelecido na academia, uma vez que se estuda para tratar e curar as pessoas, e pouco sobre uma boa relação e comunicação entre as pessoas, podendo ser este um dos problemas identificados, a partir deste estudo. Desse modo, sugere-se que sejam estabelecidos temas como estes dentro dos cursos de graduação na possibilidade de capacitar ou habilitar estes futuros profissionais de saúde, uma vez que a comunicação tendo sido considerada uma ferramenta complexa no processo de adoecimento, em especial entre profissionais e pessoa doente e família.

Por fim, este estudo possui como limitação o questionamento aos participantes apenas na fase de diagnóstico da doença, não sendo investigado a relação entre profissionais, pacientes, familiares e/ou cuidadores ao longo do processo de adoecimento, podendo esta ter sido melhor construída posteriormente.

\section{REFERÊNCIAS}

1. Organização Mundial da Saúde. Câncer>Perfil de países com câncer em 2020. Genebra: WHO; 2020. [acesso em 12 jul. 2020]. Disponível em:

https: / /www.who.int/cancer/countr y-profiles/en/

2. Brasil. Instituto Nacional de Câncer. Estatísticas de Câncer. Brasília: Ministério da Saúde; 2020. [acesso em 12 jun. 2020]. Disponível em: 
https://www.inca.gov.br/numerosde-cancer.

3. Moore PM, Rivera S, Bravo-Soto GA, Olivares C, Lawrie TA. Treinamento de habilidades de comunicação para profissionais de saúde que trabalham com pessoas com câncer. Cochrane Database of Systematic Reviews; 2018.

4. Neves FB, Noguez PT, Guimarães SRL, Muniz RM, Pinto BK. Decisões contraditórias: motivos que levam o familiar cuidador a omitir 0 diagnóstico de câncer. Rev Enferm UFPE On Line. 2017; 11(2):591-600.

5. Tong A, Sainsbury P, Craig J. Consolidated criteria for reporting qualitative research (COREQ): a 32item checklist for interviews and focus groups. Int J Qual Health Care. 2007; 19(6):349-57.

6. Fontanella BJB, Ricas J, Turato ER. Amostragem por saturação em pesquisas qualitativas em saúde. Cad Saúde Pública. 2008; 24(1):17-27.

7. Souza FN, Costa AP, Moreira A. Análise de dados qualitativos suportada pelo Software webQDA. In: VII Conferência Internacional de TIC na Educação: Perspectivas de Inovação; 2011 mai 49-56; Braga.
8. Braun V, Clarke V. Using thematic analysis in psychology. Qual Res Psychol. 2006; 3(2):77-101.

9. Bueno IAF, Tarabay CH, Cruz LMT. Comunicação em oncologia e ajustamento psicológico: uma revisão de literatura. Psicol Saúde Doenças. 2016; 17(3):527-541.

10. Bastos BR, Fonseca ACG, Pereira AKS, Silva LCS. Formação dos profissionais de saúde na comunicação de más notícias em cuidados paliativos oncológicos. Rev Bras Cancerol. 2016; 62(3):263-6.

11. Aoun SM, Breen LJ, Oliver DJ, Henderson RD, Edis R, O'Connor M, et al. Family carers' experiences of receiving the news of a Motor Neurone Disease: a national survey. J Neurol Sci. 2017; 372:144-151.

12. Eun KC, Duarte ME, Hyemon $S$, Wanda P, Julie AK. Communicating Bad News: Insignts for the Design of Consumer Health Technologies. JMIR Hum Factors. 2019; 6(2):e8885.

13. José SAP, Carmo SA, Vieira LR, Cardoso LCP, Ferrari SR, Pinto ACR, et al. A comunicação de notícias difíceis sobre a abordagem aplicada à oncologia: uma revisão bibliométrica. Res Soc Dev. 2020; 9(9):e569997570. 
14. Gilligan T, Coyle N, Frankel RN, Berry DL, Bohlke K, Epstein RN, et al. Patient-Clinician Communication: American Society of Clinical Oncology Consensus Guideline. J Clin Oncol. 2017; 35(31):3618-3632.

15. Silva SCB, Guedes MR. Percepções dos acompanhantes de pacientes em estado de terminalidade. REFACS (online). 2017; 5(2):221-227.

16. Costa RSL, Lima RSM, Félix TC, Mota TMSC, Tavares EA, Queiroz GJC, et al. Sentimentos e expectativas de mulheres frente ao diagnóstico de câncer de mama. J Health NPEPS. 2020; 5(1):290-305.

17. Santos RP, Pereira BC, Fava SMCL, Resck ZMR, Dázio EMR. Fatores contextuais da comunicação do diagnóstico de câncer no processo de finitude e morte. Enferm Brasil. 2018; 17(2):134-140.

18. Fontes CMB, Menezes DV, Borgato MH, Luiz MR. Communicating bad news: an integrative review of the nursing literature. Rev Bras Enferm. 2017; 70(5):1089-95.

Financiamento: Os autores declaram que não houve financiamento.

Conflito de interesses: Os autores declaram não haver conflito de interesses.

\section{Participação dos autores:}

- Concepção: Barboza MCN, Muniz RM, Cagliari R, Rockembach JA, Vasconcellos SA, Dias LV.

- Desenvolvimento: Barboza MCN, Muniz RM, Cagliari R, Rockembach JA, Vasconcellos SA, Dias LV.

- Redação e revisão: Barboza MCN, Muniz RM.

Como citar este artigo: Barboza MCN, Muniz RM, Cagliari R, Rockembach JA, Vasconcellos SA, Dias LV. Comunicação do diagnóstico de câncer colorretal à pessoa e família e/ou cuidador. J Health NPEPS. 2020; 5(2):226-239.

Submissão: 01/06/2020

Aceito: $10 / 09 / 2020$

Publicado: 04/12/2020 